\title{
Investigation of Four Wave Mixing Effect at Different Transmission Power Levels and Channel Spacing
}

\author{
Mehtab Singh \\ Electronics Technology Department \\ Guru Nanak Dev University \\ Amritsar
}

\author{
Vishal Sharma \\ Electronics Technology Department \\ Guru Nanak Dev University \\ Amritsar
}

\begin{abstract}
There is an incredible focus on broadband fiber optical transmission system as it offers many advantages over other communication systems such as large bandwidth and significantly low losses [1]. This high bandwidth in optical communication networks is further increased by using Wavelength Division Multiplexing. In WDM based fiber optical communication systems nonlinearities in fiber are a limiting factor which degrade the system performance and limit the data rate and bandwidth of the system [3]. As we increase the transmission power levels so as to transmit data for long-haul communications systems these non linear effects tend to accumulate. Due to these non linear effects, all input signals may interact with each other in a complicated way which degrades overall performance of optical communication system. In WDM systems, the interaction of input signals with each other adversely affects the performance of the system. Hence it is of great importance to understand fiber non linear effects and its effect on optical fiber communication system. The main objective of this paper is to study non linear effects in fiber optic communication system mainly Four Wave Mixing (FWM) and to analyze to effect of increasing optical transmission powers and channels spacing in a wave division multiplexing system.
\end{abstract}

The results show that as the transmission power is increased, the efficiency of four wave mixing effect increases. Also if the channel spacing in a WDM system is kept uneven, the power levels of mix frequencies produced as a result of FWM effects is reduced to a considerable amount.

\section{General Terms}

Investigation, Four Wave Mixing, Wave Division Multiplexing

\section{Keywords}

Wave Division Multiplexing (WDM), Four Wave Mixing (FWM), channel spacing, transmission power level, non linear effects

\section{INTRODUCTION}

The phenomenon of interaction between two or more wavelengths to produce more wavelengths due to scattering of photons incident on the fiber is known as four wave mixing (FWM) also named as four-photon mixing [1],[4],[7]. It is one of the most crucial parameter which determines the performance of an optical transmission system. It is observed that two or more information caring optical signals travelling in the same fiber interact with each other very weakly. However over long-haul transmission, these weak interactions become very significant. The main reason of four wave mixing in optical fiber is the change of refractive index of optical fiber with optical power. Four wave mixing can also be considered as
Kerr effect in optical domain [7]. Chromatic dispersion and fiber non linear effects pose a major restriction on maximum repeater distance and bandwidth of fiber optic communication system. The interactions between information carrying optical signal and optical fiber can lead to signal interference, signal distortion, scintillations and distortion of information carrying signal which degrades system's performance [2], [3]. There are two categories of fiber nonlinearities. One amongst them is stimulated scattering (Raman and Brillion) which results in intensity depending gain and loss as a result of stimulated process. In Stimulated Raman Scattering (SRS), light interact with vibrating silica molecules which causes conversion of frequency of light and results in attenuation of shorter wavelengths in a WDM system. In Stimulated Brillion Scattering (SBS), light interact with sound wave in fiber which results in frequency conversion and results in reversal of the direction of light propagating in the fiber. The other types of non linear effects are Four Wave Mixing (FWM), Self-Phase Modulation (SPM) and Cross-Phase Modulation (XPM). In Self Phase modulation (SPM), there is an interaction between intensity of the light wave and optical phase of the same light wave due to non linear refractive index of the fiber whereas in Cross Phase Modulation (XPM), there is an interaction between intensity of a light signal with optical phase of another light signal carrying along with the signal in same fiber due to non linear refractive index of the fiber. Four wave mixing is a third order distortion of signal in which two or more nearby frequency travelling in the same fiber interact with each other and as a result produce new frequencies also known as beat frequencies which travel along the original signal travelling in the fiber. As a result of FWM in a multi channel system several impairments occur in the transmission process such as crosstalk and inter symbolic interference (ISI) which degrades the overall performance of the system [5]. In this investigative paper, analysis has been done to determine the effect of increasing optical transmission power of all the channel in a WDM system on the efficiency of FWM effect in by analyzing input/output spectrum of signals and BER of the signals using Optisystem simulation software. Also analysis has been done to determine the performance of FWM effect when unequal channel spacing is considered in a WDM optical transmission system.

The rest of the paper is organized as follows; in section 2, mathematical analysis of four wave mixing is discussed in detail. In section 3, simulation setup and different simulation parameters used in this analysis is discussed. In section 4 , results of all the simulations are discussed in detail and in section 5, the conclusion of this analytical study is given and discussed. 


\section{MATHEMATICAL ANALYSIS}

One of the main reasons of signal degradation in an optical communication system is intermodal dispersion. By use of a single mode fiber to carry information signal, intermodal dispersion can be eliminated. Yet the single mode travelling in an SMF does not consist of a single wavelength rather a group of wavelengths as a result of finite spectral width of transmitting source. Each wavelength travels with different velocity as speed of light is dependent on the refractive index on fiber which depends upon the wavelength of optical signal carrying information [6], [9]. As a result a wavelength dependant group velocity delay is produced. This is known as intramodal or modal dispersion and can be minimized by using dispersion compensating fiber (DCF), fiber bragg grating (FBG), electronic dispersion compensation (EDC) techniques and digital filters. In four wave mixing two or more information carrying signals travelling in the same fiber can interact with each other to produce new signals at different wavelengths due to dependence of refractive index of silica fiber on intensity of light carrying signal which travels at the expense of original information carrying signal and thus performance of the system is degraded.

If three information carrying signal with frequencies $f_{a}, f_{b}$ and $f_{c}$ are incident at the input of fiber, then new beat frequencies are generated whose frequencies is given by [8]:

$$
f_{a b c}=f_{a}+f_{b}-f_{c} \quad \text { where, }(\mathrm{a}, \mathrm{b}, \mathrm{c}=1,2,3)
$$

There is increase in number of side bands due to FWM effect and is given by [8]:

$$
\mathrm{M}=\left(\frac{N^{3}-N^{2}}{2}\right)
$$

Where $\mathrm{N}$ signifies the number of channels available and $\mathrm{M}$ denotes total number of side bands generated. For example, nine (9) channels produce 324 side bands. Fig. 1 demonstrates how FWM mixing products due to channel increases.

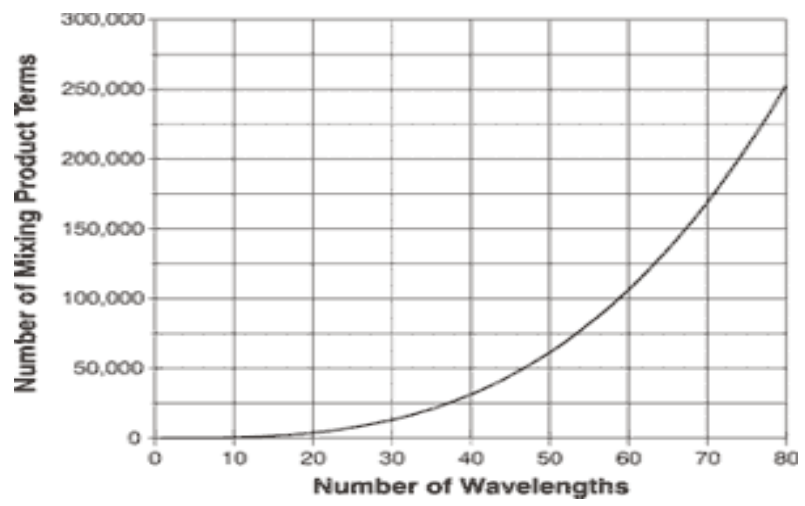

Fig. 1 Number of four wave mixing products [6]

The FWM power produced at end of optical fiber interaction of frequencies $f_{a}, f_{b}$ and $f_{c}$ is given by [11]:

$$
P_{F W M}=\frac{1024 \pi^{2}}{n^{4} \lambda^{2} c^{2}}\left[\frac{D \chi_{111 L_{e f f}}}{A_{e f f}}\right]^{2} P_{a} P_{b} P_{c} e^{-\alpha L} \eta
$$

Where $\mathrm{n}$ is refractive index of the fiber, $\lambda$ is the wavelength of information carrying signal, D is degeneracy factor and $P_{a}, P_{b}, P_{c}$ are power values of three input waves. $A_{e f f}$ is the effective area, $L_{e f f}$ is the effective length, $\eta$ is the mixing efficiency.

The efficiency of FWM is given by [10]:

$$
\eta=\frac{\alpha^{2}}{\alpha^{2}+\Delta \beta^{2}}\left(1+\frac{4 e^{-\alpha l} \sin ^{2}\left(\frac{\Delta \beta}{2}\right)}{\left(1-e^{-\alpha L}\right)^{2}}\right)
$$

Where $\Delta \beta$ is propagation coefficient and can be written as [10]:

$$
\Delta \beta=\beta_{a b c}+\beta_{c}-\beta_{b}-\beta_{a}
$$

Here, $\beta$ denotes propagation constant of light.

\section{SIMULATION SETUP}

The main aim of this investigative study is to analyze how channel spacing in wave division multiplexing (WDM) and chromatic dispersion coefficient affects the efficiency of four wave mixing (FWM) in optical fiber transmission system. The objective is to use the outcomes of the simulations to establish a feasible hardware implementation. Simulations are performed using Optisystem 7.0 simulation software with a 4 channel WDM system using externally modulated laser sources on an optical fiber link of length $10 \mathrm{~km}$ at $1550 \mathrm{~nm}$ central wavelength. The simulation model used in the analysis shown in Fig. 3 and Optisystem simulation setup is shown in Fig. 4.

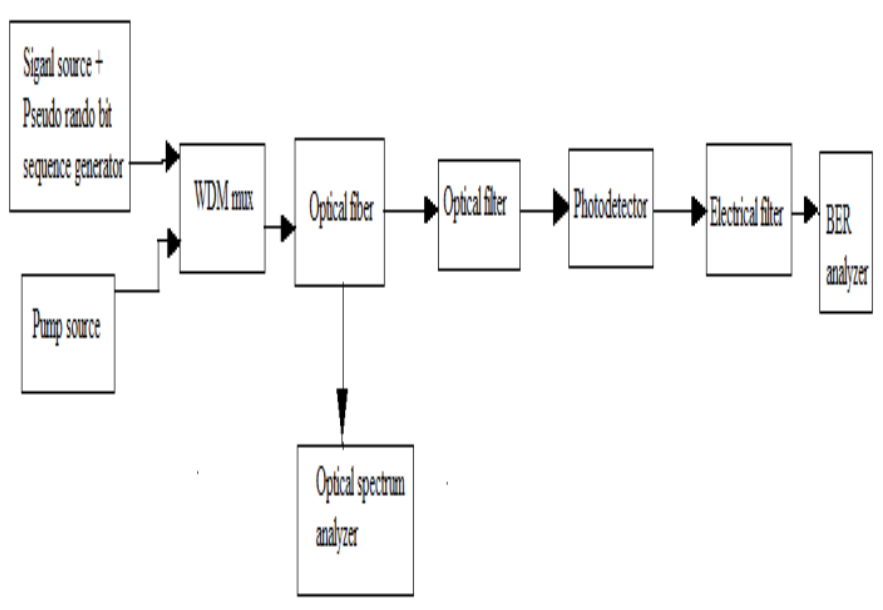

Fig. 2 Simulation model [4] 


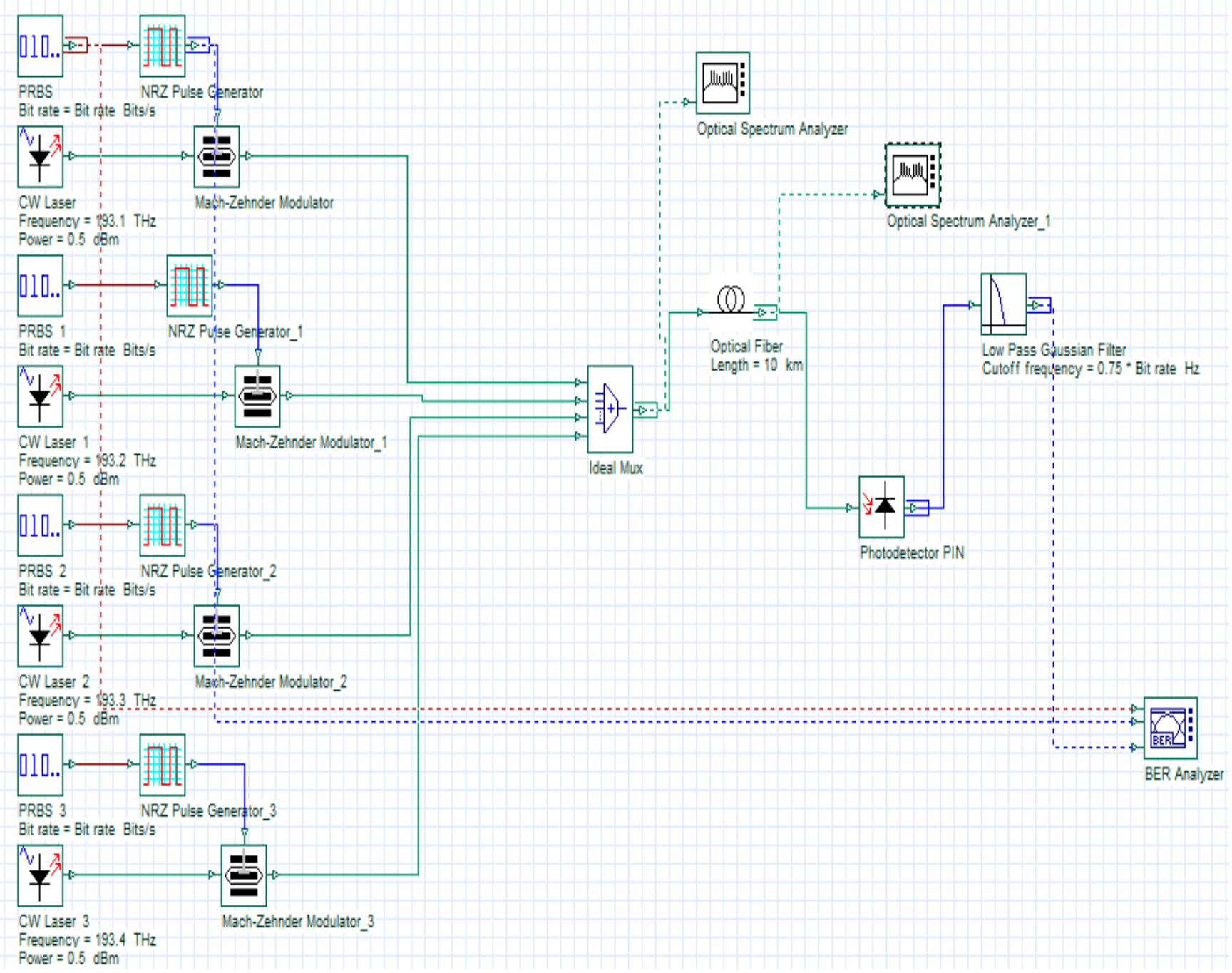

Fig. 3 Simulation setup

The transmitter section consists of a PRBS which generates a stream of 0 's and 1's at a rate of $2.5 \mathrm{Gbps}$ which are directed to the input of NRZ pulse modulator. NRZ pulse modulator converts these 0 's and 1's in form of electric pulses having NRZ format. These electrical pulses from output of NRZ pulse modulator are directed to input of Mach-Zender modulator which modulates information signal with a $\mathrm{CW}$ laser. The transmitter section consists of 4 such channels separated at some frequency interval. In this investigative study 4 WDM channels have been considered which are separated from each other at some frequency interval. These 4 channels are multiplexed and directed into a SMF of length $10 \mathrm{~km}$. The optical signal from the output of the optical fiber is directed to the input of PIN photodiode which converts optical signal to an electrical signal. An APD photodiode can also be considered in place of PIN photodiode for better results. Output of PIN photodiode is directed to low pass Gaussian filter which eliminates all the high frequency noise present in the received electrical signal. At the last stage, received signal is analyzed using BER analyzer.

\section{RESULTS AND DISCUSSIONS}

In this paper, analysis has been done to measure the effect of increasing optical transmitting power on the efficiency of four wave mixing (FWM) phenomenon in a WDM optical transmission system. Also investigation has been done to analyze how efficiency of FWM effect behaves when unequal channel spacing is used in WDM system. Various simulation and analyses were performed using Optisystem 7.0 software and using above presented simulation setup. Different simulation parameters are presented in table no.1. Results have been evaluated on the basis of eye diagrams and input/output frequency spectrum of the signals.

Table No. 1 Simulation Parameters

\begin{tabular}{|c|c|c|}
\hline $\begin{array}{c}\text { Serial } \\
\text { No. }\end{array}$ & Parameters & Value \\
\hline 1. & Reference Frequency $(\mathrm{nm})$ & 1550 \\
\hline 2. & Fiber Length $(\mathrm{km})$ & 10 \\
\hline 3. & Dispersion Coefficient $(\mathrm{ps} / \mathrm{nm} / \mathrm{km})$ & 4 \\
\hline 4. & Differential group delay $(\mathrm{ps} / \mathrm{km})$ & 0.5 \\
\hline
\end{tabular}




\begin{tabular}{|c|c|c|}
\hline 5. & Effective Area (um²) & 50 \\
\hline 6. & Attenuation (dB) & 0.2 \\
\hline 7. & Laser Frequency (THz) & $193.1-193.4$ \\
\hline 8. & Filter Type & Gaussian \\
\hline 9. & Modulator Type & Mach-Zender \\
\hline 10. & Extinction Ration (dB) & 30 \\
\hline 11. & Symmetric Factor & -1 \\
\hline 12. & Bit Rate (Gbps) & 2.5 \\
\hline 13. & Transmitting Power (dBm) & $1-12$ \\
\hline 14. & No of Samples & 8192 \\
\hline 15. & Sample Bit Rate & 64 \\
\hline
\end{tabular}

Frequency spectrum of input signal when equal spacing has been used in all the channels is shown in Fig. 4

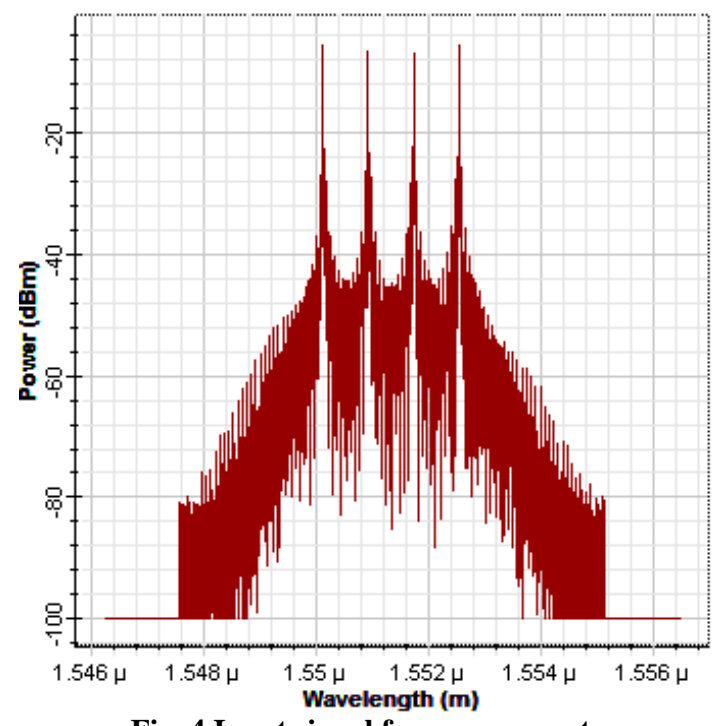

Fig. 4 Input signal frequency spectrum

It can be seen from the frequency spectrum of the input signal that there are 4 channels which are equally spaced and there are no other frequency components present in the spectrum of the input of WDM transmission signal.The spectrum of output signals when transmitting power is 0.5 $\mathrm{dBm}, 4 \mathrm{dBm}, 8 \mathrm{dBm}$ and $12 \mathrm{dBm}$ is shown in Fig. 5, Fig. 6 . Fig. 7 and Fig. 8 respectively.

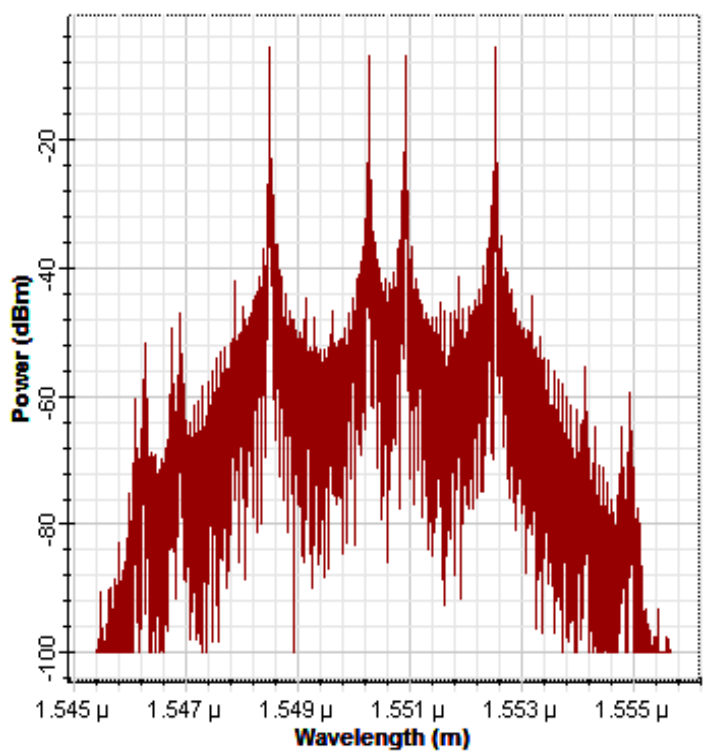

Fig. 5 Output signal Frequency spectrum $(P=0.5 \mathrm{dBm})$

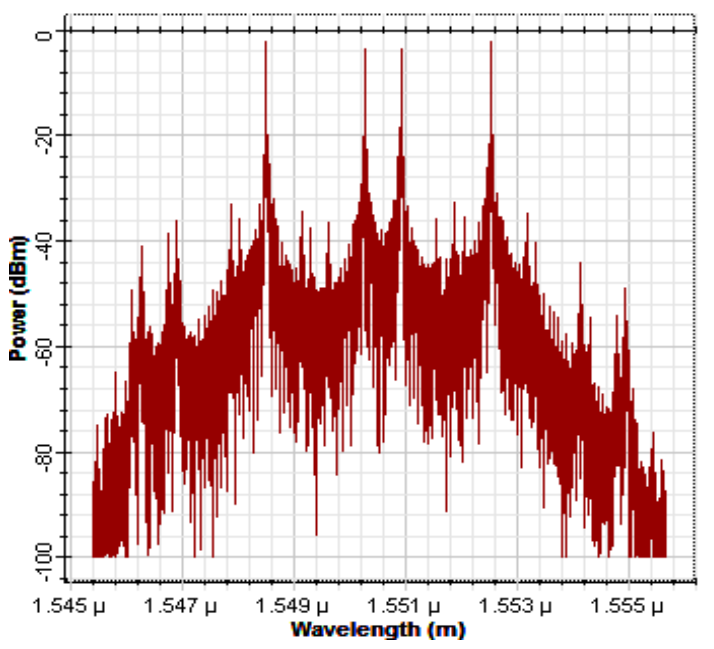

Fig. 6 Output signal Frequency spectrum $(P=4 \mathrm{dBm})$

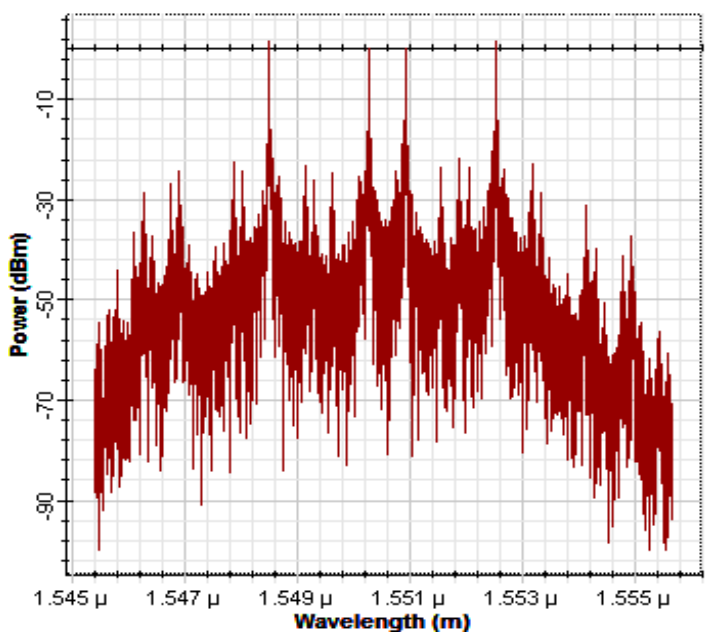

Fig. 7 Output signal Frequency spectrum $(P=8 \mathrm{dBm})$ 


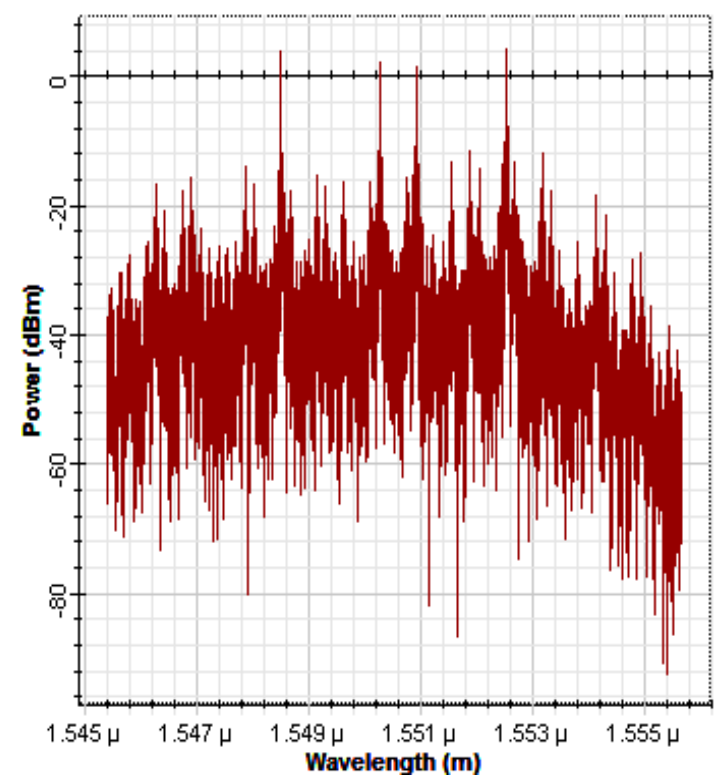

Fig. 8 Output signal Frequency spectrum $(P=12 \mathrm{dBm})$

From the above results it can be seen that as the optical transmitting power is increased the power level of beat frequencies produced as a result of FWM effect increases and hence overall performance of WDM transmission system decreases. In this paper, the impact of channel spacing on the efficiency of FWM effect has also been analyzed by evaluating eye diagrams and frequency spectrum for both equally and unequally spaced channels. Fig. 9 and Fig. 10 shows frequency spectrum and eye diagram of output signal when equal channel spacing is considered for a transmitting power of $0.5 \mathrm{dBm}$.

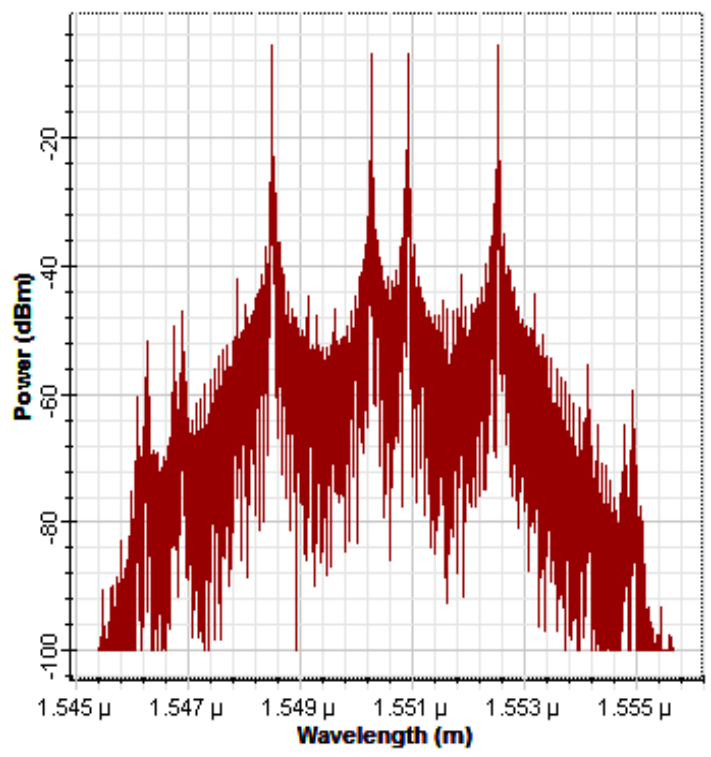

Fig.9 Output signal Frequency spectrum with equal channel spacing $(P=0.5 \mathrm{dBm})$

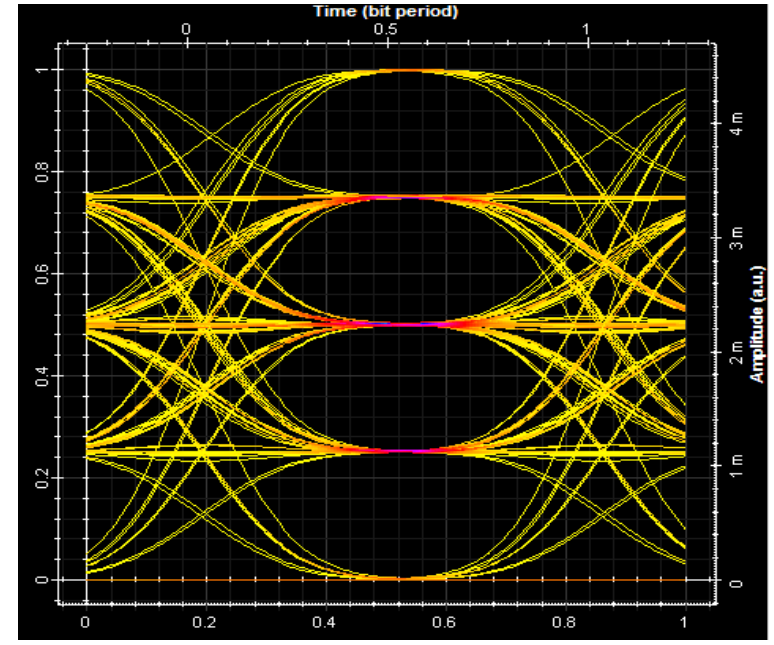

Fig.10 Eye Diagram of received signal with equal channel spacing $(P=0.5 \mathrm{dBm})$

Fig. 11 and Fig. 12 shows frequency spectrum and eye diagram of output signal when unequal channel spacing is considered for a transmitting power of $0.5 \mathrm{dBm}$.

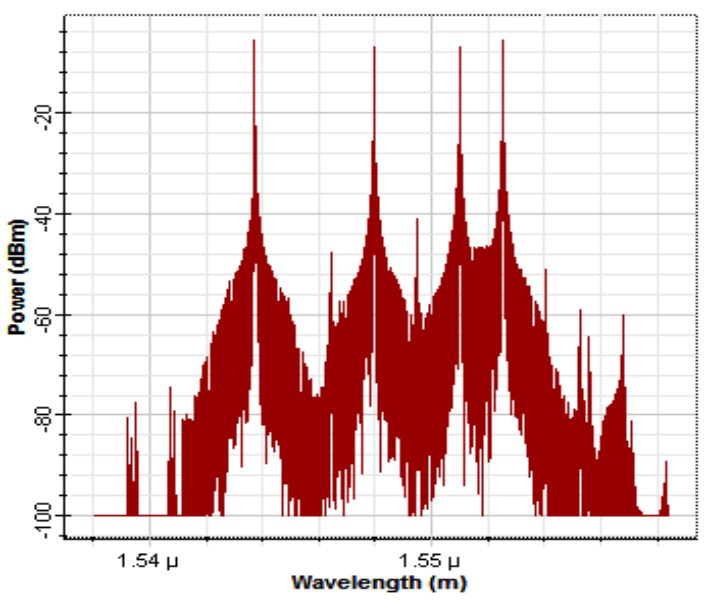

Fig.11 Output signal Frequency spectrum with unequal channel spacing $(P=0.5 \mathrm{dBm})$

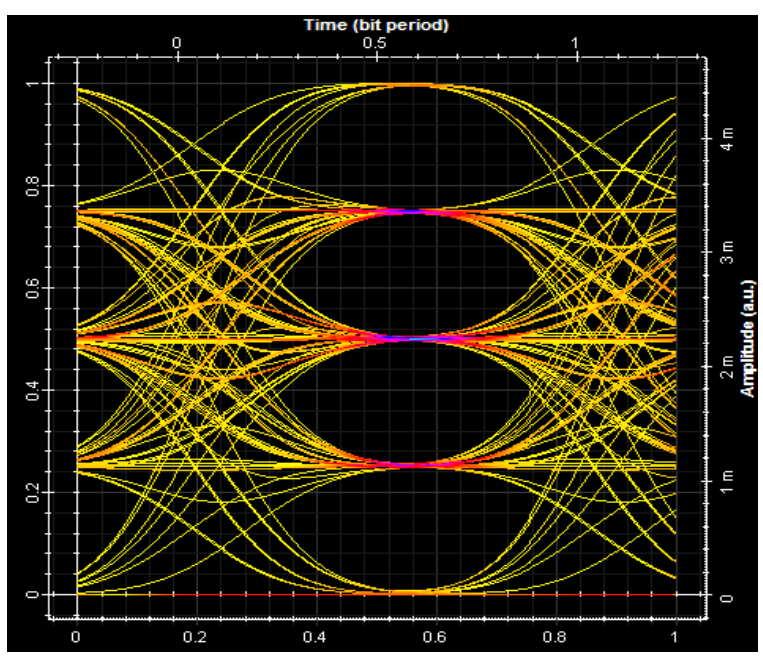

Fig.12 Eye Diagram of received signal with unequal channel spacing $(P=0.5 \mathrm{dBm})$ 
It can be seen from above presented results that when unequal channel spacing is used in WDM optical transmission system, system shows a better performance as compared to equal spacing channels WDM system.

\section{CONCLUSION}

In this paper investigation has been done to analyze the efficiency of four wave mixing (FWM) effect when the optical signal transmitting power is increased gradually. From the above presented results it can be concluded that as the optical signal transmission power level is increased the effect of four wave mixing increases as the power level of beat frequencies increases with increase in optical signal power levels. Also analysis has been done to analyze performance of FWM when unequal channel spacing is considered. It can be concluded from the results that when unequal channel spacing was considered, the power levels of beat frequencies generated as a result of FWM effect in WDM system were suppressed to a great extent and thus the performance of optical WDM system is enhanced. Therefore to improve overall performance of an optical WDM transmission system, unequal spacing in channels is recommended along with an optimum value of optical power transmission levels.

\section{REFERENCES}

[1] O. Aso , M. Tadakuma, and S. Namiki, "Four-wave mixing in optical fibers and its applications", Furukawa Review, no. 19, pp. 63.68, 2000.

[2] P. L. Li, D. X. Huang, X. L. Zhang, J. Chen, and L. R. Huang," Theoretical Analysis of Tunable Wavelength Conversion Based on FWM in a Semiconductor Fiber Ring Laser," IEEE Journal of quantum electronics, vol. 41, no. 3, pp. 581-588, March 2005.

[3] G.charlet, E.corbel, J.Lazaro, A.klekamp, W.Idler, R.Dischler, S.Bigo "comparision ofsystem performance at $50,62.5$ and $100 \mathrm{GHz}$ channel spacing over transoceanic distances at $40 \mathrm{Gcit} / \mathrm{s}$ channel rate using
RZDPSK” Electronics Letters 3rd, vol.41, no.3, February 2005.

[4] Osamu Aso, Masateru Tadakuma and Shu Namiki," Four-Wave Mixing in Optical Fibers and Its Applications", WP Team, Opto-technology Lab., $R \& D$ Div.

[5] G. P. Agrawal, "Nonlinear Fiber Optics, Second Edition", Academic Press, San Diego, USA, Chap. 10, 1995.

[6] Yamamoto, Optical Fiber Communications Technology, Nikkan Kogyo Shimbun, Chap.11, 1995.

[7] Edmund Kueh Boon Kiang, S. P. Majumder, "Analytical Evaluation of the Impact of Four Wave Mixing on the Bit Error Rate Performance of a WDM Soliton Transmission Link", Journal of Optical Communications, vol. 23, no. 4, pp. 122-126, 2002.

[8] J. Zweck and C. R. Menyuk, "Reduction of intrachannel four-wave mixing using subchannel multiplexing”, IEEE Photonics TechnologyLetters, vol. 15 , no. 2, pp. 323-325, 2002.

[9] M. Kowalewski, M. Marciniak and A. Sedlin, "Nonlinear interactions in wavelength-multiplexed optical fibre telecommunication systems", J. Opt. A: Pure Appl. Opt. 2 pp.319-326. 2000.

[10] S. Shuxian, "Higher-order four-wave mixing and its effect in WDM systems," Optical Society of America, vol. 7, no. 4, pp 166-171, 2000.

[11] H. G. Batshon, I. B. Djordjevic, and B. V. Vasic, "An improved technique for suppression of intrachannel four-wave mixing in $40-\mathrm{Gb} / \mathrm{s}$ optical transmission systems", IEEE Photonics Technology Letters, vol. 19, no. 2, pp. 67-72, 2007. 